\title{
A Coronary Artery Calcium Score of Zero in Patients Who Have Undergone Coronary Computed Tomography Angiography Is Associated With Freedom From Major Adverse Cardiovascular Events
}

\author{
Yuhei Shiga $^{\text {a, c }}$, Joji Morii ${ }^{a}$ c, Yoshiaki Idemoto ${ }^{a}$, Kohei Tashiro ${ }^{a}$, Yuiko Yano ${ }^{\text {a }}$ \\ Yuta Kato $^{\text {a }}$, Takashi Kuwano ${ }^{\text {a, }}$ Makoto Sugihara ${ }^{\text {a }}$, Shin-ichiro Miura ${ }^{\text {a, b, d }}$
}

\begin{abstract}
Background: The coronary artery calcification score (CACS) is a good marker of future cardiovascular risk. We determined the association between the CACS and the prognosis in patients who have undergone coronary computed tomography angiography (CCTA).

Methods: We performed a prospective cohort study and enrolled 502 consecutive patients who underwent CCTA for screening of coronary artery disease (CAD) at Fukuoka University Hospital (FUCCTA Registry) and either were clinically suspected of having CAD or had at least one cardiovascular risk factor with a follow-up of up to 5 years. The patients were divided into CACS $=0$ and CACS > 0 groups. Using CCTA, $\geq 50 \%$ coronary stenosis was diagnosed as $\mathrm{CAD}$, and the number of significantly stenosed coronary vessels (VD), Gensini score and CACS were quantified. The primary endpoint was major adverse cardiovascular events (MACE: cardiovascular death, ischemic stroke, acute myocardial infarction and coronary revascularization).
\end{abstract}

Results: \%CAD, the number of VD and the Gensini score in the $\mathrm{CACS}=0$ group were significantly lower than those in the CACS $>$ 0 group. $\% \mathrm{MACE}$ in the $\mathrm{CACS}=0$ group was also significantly lower than that in the CACS $>0$ group. Kaplan-Meier curves indicated that the CACS $=0$ group showed significantly greater freedom from MACE than the CACS $>0$ group $(\mathrm{P}=0.008)$. Finally, only CACS $=$ 0 was independently associated with MACE (odd ratio: $0.41,95 \%$ confidence interval: $0.17-0.97, \mathrm{P}=0.041$ ).

Manuscript submitted August 28, 2020, accepted September 3, 2020

Published online September 21, 2020

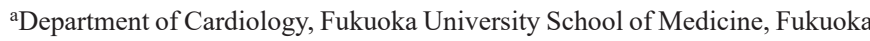
814-0180, Japan

bDepartment of Cardiology, Fukuoka University Nishijin Hospital, Fukuoka 814-8522, Japan

${ }^{\mathrm{c}}$ These authors contributed equally to this manuscript.

${ }^{\mathrm{d} C}$ Corresponding Author: Shin-ichiro Miura, Department of Cardiology, Fukuoka University School of Medicine, 7-45-1 Nanakuma, Jonan-ku, Fukuoka 814-0180, Japan.Email: miuras@cis.fukuoka-u.ac.jp

doi: https://doi.org/10.14740/jocmr4335
Conclusions: A CACS of 0 in patients who underwent CCTA was associated with a good prognosis.

Keywords: Coronary artery disease; Coronary artery calcification; Coronary computed tomography angiography; Major adverse cardiovascular events

\section{Introduction}

The coronary artery calcium score (CACS) plays an important role in cardiovascular disease (CVD) risk stratification, and is significantly associated with the occurrence of major CVD events [1-5]. CACS is a strong predictor of coronary artery disease (CAD) [1]. Among all negative CVD risk markers, CACS of 0 was shown to be the strongest marker of CVD [2]. A CACS of 0 in patients undergoing coronary computed tomography angiography (CCTA) for suspected stable angina has a high negative predictive value for the exclusion of obstructive CAD and is associated with a good medium-term prognosis [3].

Thus, CACS is a good marker of future CVD risk. We have been studying the CCTA Registry at Fukuoka University in Japan (FU-CCTA Registry) and identified CVD risk factors for the primary prevention of CAD [6-11]. Using the FUCCTA Registry, we determined the association between CACS and the prognosis in patients who underwent CCTA.

\section{Materials and Methods}

\section{Study subjects}

We performed a prospective cohort study and enrolled 502 consecutive patients who underwent CCTA for screening of coronary atherosclerosis at Fukuoka University Hospital (FUCCTA Registry) and either were clinically suspected of having CAD or had at least one cardiovascular risk factor with a follow-up. All subjects underwent CCTA between 2011 and 2016. Patients with age $\geq 20$ years regardless of gender 
were enrolled. Patients with creatinine $>2.0 \mathrm{mg} / \mathrm{dL}$, severe liver dysfunction or contrast-induced allergy were excluded from the study. The patients were divided into CACS $=0$ and CACS $>0$ groups. We determined major adverse cardiovascular events (MACE: cardiovascular death, ischemic stroke, acute myocardial infarction and coronary revascularization) as a primary endpoint in patients with a follow-up of up to 5 years (average: $3.5 \pm 0.6$ years). This study was conducted in compliance with the ethical standards of the responsible institution on human subjects as well as with the Helsinki Declaration. The protocol in this study was approved by the Ethics Committee of Fukuoka University Hospital, and all subjects gave their written informed consent to participate.

\section{Evaluation of coronary stenosis, the number of signifi- cantly stenosed coronary vessels (VD), Gensini score and CACS using CCTA}

We evaluated coronary stenosis using CCTA as previously described [6-11]. Patients were assessed by 64-multidetector computed tomography (MDCT) on an Aquilion 64 (TOSHIBA, Tokyo, Japan) or 320-MDCT on an Aquilion ONE ViSION (TOSHIBA, Tokyo, Japan). Fifteen coronary artery segments were assessed in all patients. Narrowing of the normal contrast-enhanced lumen to $\geq 50 \%$ that could be identified in multiplanar reconstructions or cross-sectional images was defined as significant stenosis. When patients had significant coronary stenosis, they were initially diagnosed with CAD. In addition, in all patients, the severity of CAD was assessed in terms of the VD and the Gensini score [12]. CAC was defined on CT images as the presence of more than two contiguous pixels with greater than 130 Hounsfield Units. The CACS in each lesion was then computed by the Agatston method [13]. When the patients had significant coronary stenosis as assessed by CCTA and received coronary intervention immediately after CCTA, the intervention was not included in MACE as coronary revascularization.

\section{Assessment of risk factors for CAD}

Systolic blood pressure (SBP), diastolic BP (DBP), serum levels of triglyceride (TG), high-density lipoprotein cholesterol (HDL-C), low-density lipoprotein cholesterol (LDL-C), fasting blood glucose (FBG), hemoglobin A1c (HbA1c), smoking status, and family history (myocardial infarction, angina pectoris or sudden death) were collected as risk factors in all patients. All of the blood samples were drawn in the morning after the patients had fasted overnight. To measure the visceral fat area (VFA), a CT scan was also performed. The value was measured from CT cross-sectional scans at the level of the umbilicus with a workstation on a Ziostation (Ziosoft Inc., Tokyo. Japan). The characteristics of patients were obtained from medical records with regard to history of hypertension (HTN), dyslipidemia (DL), diabetes mellitus (DM) and smoking. Information on medication use was obtained from medical records and included angiotensin II receptor blocker/angioten- sin-converting-enzyme inhibitor (ARB/ACEI), calcium channel blocker (CCB), $\beta$-blocker, diuretic, statin, sulfonylurea, biguanide, dipeptidyl peptidase-4 inhibitor (DPP-4I) and insulin.

\section{Statistical analysis}

A statistical analysis was performed using Excel 2016 (SSRI, Tokyo, Japan) and the Stat View statistical software package (Stat View 5; SAS Institute Inc., Cary, NC, USA) Continuous variables are shown as the mean \pm standard deviation. Categorical and continuous variables were compared between the groups by a Chi-square analysis and $t$-test, respectively. A Kaplan-Meier analysis (log-rank test) was applied to verify the time-dependent occurrence of MACE in groups stratified according to whether CACS was 0 or $>0$. A multivariate analysis was performed by a logistic regression analysis for independent variables that were related to MACE. A value of $\mathrm{P}<0.05$ was considered significant.

\section{Results}

Patient characteristics, biochemical and hemodynamic parameters and medications in the $\mathrm{CACS}=0$ and $\mathrm{CACS}>0$ groups

Table 1 shows the characteristics in the $\mathrm{CACS}=0$ and $\mathrm{CACS}>$ 0 groups. There were various significant differences in patient characteristics between the CACS $=0$ and CACS $>0$ groups. The $\mathrm{CACS}=0$ group showed a significantly younger age, lower $\%$ male, $\%$ smoking, $\%$ HTN, SBP, \%DM, \%DL, \%ARB/ $\mathrm{ACEI}, \% \mathrm{CCB}, \%$ statin, $\%$ sulfonylurea and \%DPP-4I and significantly higher levels of HDL-C than the CACS $>0$ group.

\section{$\%$ CAD, VD, CACS and Gensini score in the CACS $=0$ and CACS $>0$ groups}

The CACS $=0$ group showed a significantly lower $\% C A D, V D$, CACS and Gensini score than the CACS $>0$ group (Fig. 1a-d).

\section{$\%$ MACE in the CACS $=0$ and $\mathrm{CACS}>0$ groups}

$\% \mathrm{MACE}$ in the CACS $=0$ group $(4.5 \%)$ was significantly lower than that in the CACS $>0$ group $(11.7 \%)(\mathrm{P}=0.005)$ (Fig. 2a).

\section{Kaplan-Meier curves for freedom from MACE in the $\mathrm{CACS}=0$ and $\mathrm{CACS}>0$ groups}

Kaplan-Meier curves in Figure $2 \mathrm{~b}$ show freedom from MACE in the $\mathrm{CACS}=0$ group versus $\mathrm{CACS}>0$ group. The $\mathrm{CACS}=0$ group showed significantly greater freedom from MACE than the CACS $>0$ group $(\mathrm{P}=0.008)$. 
Table 1. Patient Characteristics and Medications in the CACS $=0$ and CACS $>0$ Groups

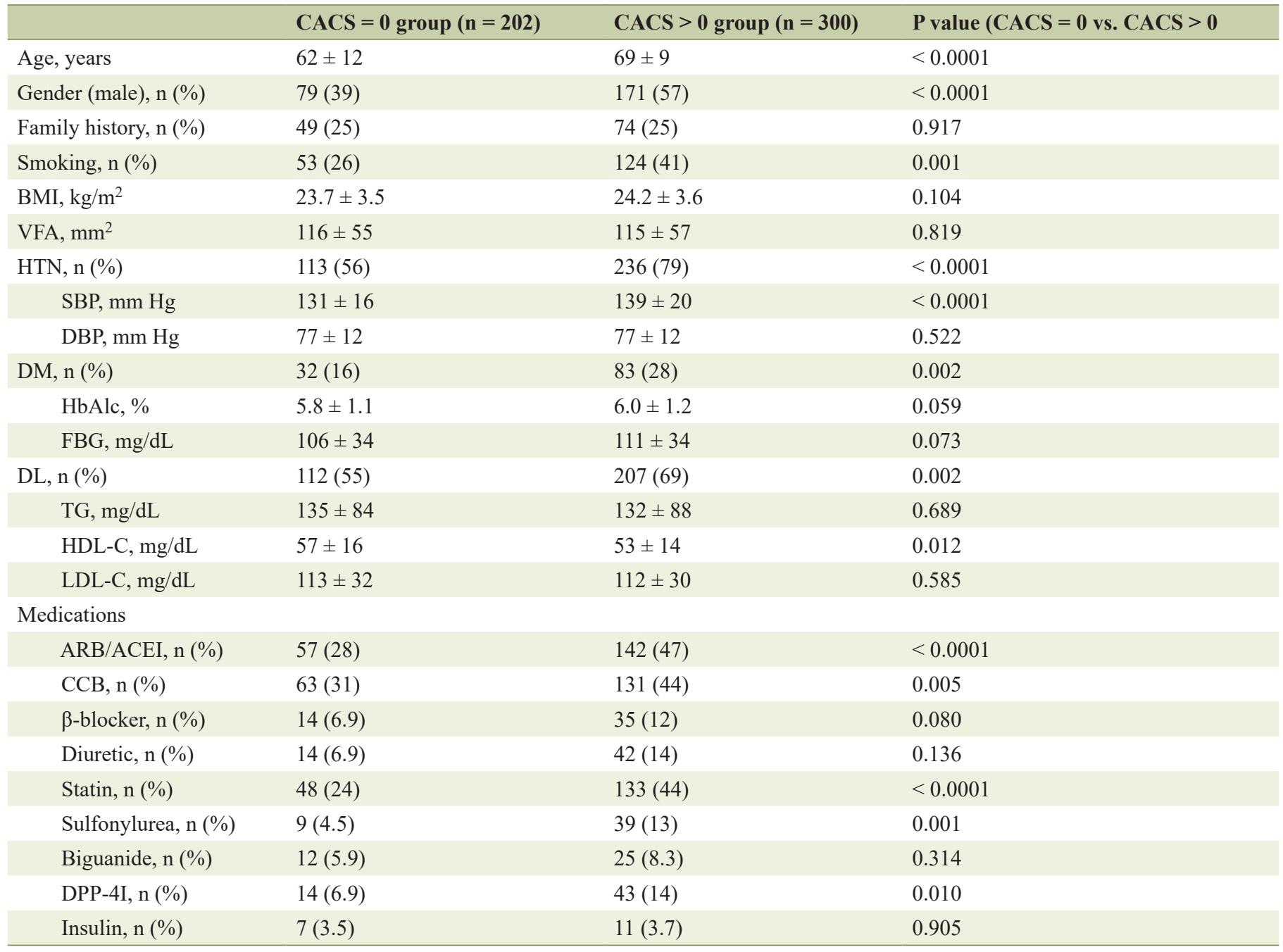

Continuous variables are expressed as mean \pm SD. BMI: body mass index; VFA, visceral fat area; HTN: hypertension; SBP: systolic blood pressure; DBP: diastolic blood pressure; DM: diabetes mellitus; HbA1c: hemoglobin A1c; FBG: fasting blood glucose; DL: dyslipidemia; TG: triglyceride; HDLC: high-density lipoprotein cholesterol; LDL-C: low-density lipoprotein cholesterol; ARB/ACEI: angiotensin II receptor blocker/angiotensin-converting enzyme inhibitor; CCB: calcium channel blocker; DPP-4I: dipeptidyl peptidase-4 inhibitor

Independent predictors of MACE by a multivariate logistic regression analysis

Table 2 shows independent predictors of MACE by a multivariate logistic regression analysis. We selected conventional risk factors (age, male, VFA, smoking, family history, HTN, $\mathrm{DL}, \mathrm{DM})$ in addition to $\mathrm{CACS}=0$ as variables. Only $\mathrm{CACS}=$ 0 was independently associated with $\operatorname{MACE}(\mathrm{P}=0.041)$.

\section{Discussion}

In the present study, using the FU-CCTA Registry, we determined the association between CACS and MACE in patients who underwent CCTA. The most important finding was that the CACS $=0$ group showed significantly greater freedom from MACE than the CACS $>0$ group. In addition, only $\mathrm{CACS}=0$ was independently associated with freedom from MACE.

We compared MACE in patients with $\mathrm{CACS}=0$ versus CACS $>0$. A CACS of 0 was the strongest marker of MACE in this study. Our results are consistent with those from previous reports $[2,3,14]$ and indicate that patients with a CACS of 0 show a good prognosis. The incidence of MACE in the present study was higher than that in previous reports $[3,14]$. Kim et al reported that the incidence of MACE in CACS $=0$ and CACS $>0$ groups was $1.3 \%$ and $4.7 \%$ over 2.8 years, respectively [14]. Wang et al reported their findings in patients who were followed-up for a median of 2.2 years [3]. The incidence of MACE was $0.6 \%$ in the CACS $=0$ group and $2 \%$ in the CACS $>0$ group. In our study, the incidence of MACE was $4.5 \%$ and $11.7 \%$ in the $\mathrm{CACS}=0$ and $\mathrm{CACS}>0$ groups, respectively, over an average of 3.5 years. It is possible that our patients 

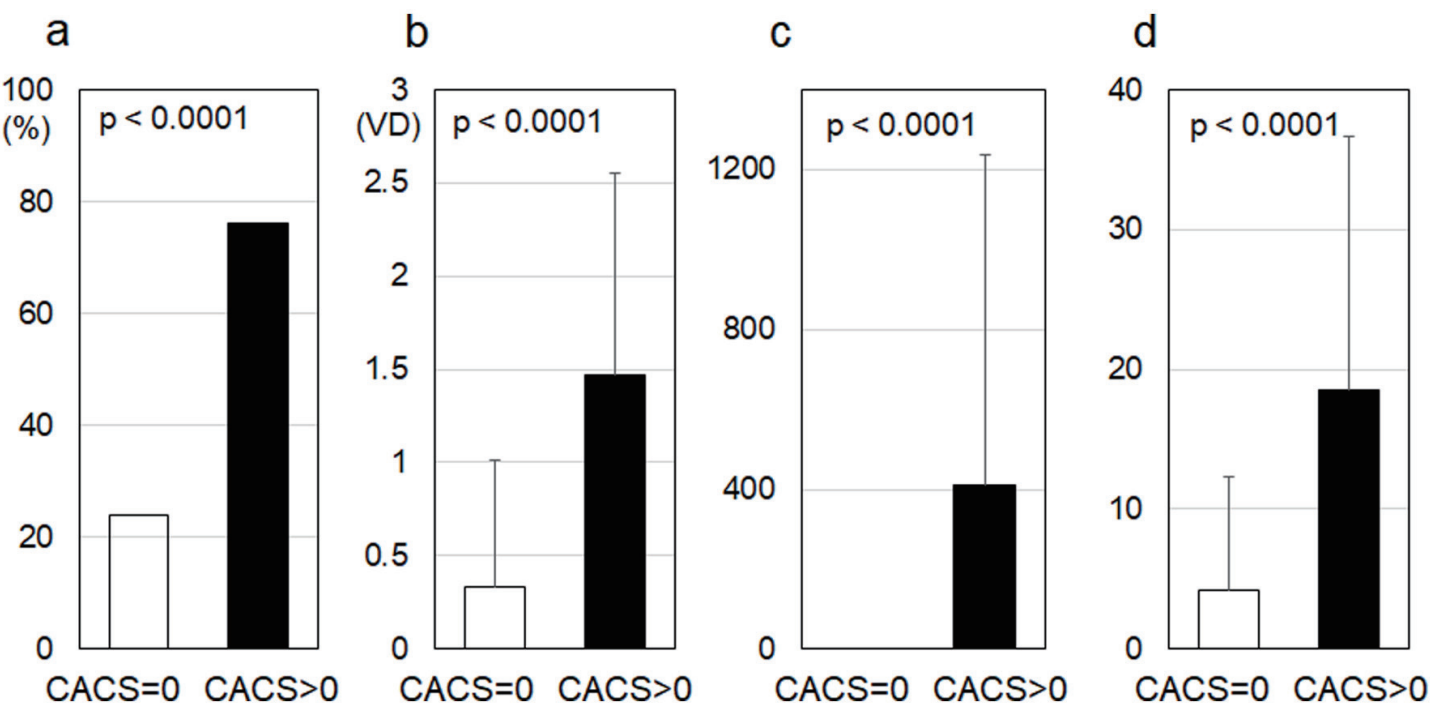

Figure 1. \%CAD (a), number of VD (b), CACS (c) and Gensini score (d) in the CACS $=0$ and CACS > 0 groups. CAD: coronary artery disease; number of VD: number of significantly stenosed coronary vessels; CACS: coronary artery calcium score.

had higher $\% \mathrm{HTN}, \% \mathrm{DL}$, and/or \%DM than those reported by Kim [14] and Wang [3]. In this study, we selected patients with higher cardiovascular risks. There is a difference between our data and theirs. After adjusting for age, gender and cardiovascular risk factors, the difference in prognostic value between $\mathrm{CACS}=0$ and $\mathrm{CACS}>0$ was no longer statistically significant [3]. On the other hand, only CACS $=0$ was independently associated with MACE in this study.

At the time of CCTA, the CACS $>0$ group had higher $\% \mathrm{CAD}$, number of VD and Gensini score than the CACS $=0$ group. When patients had significant coronary stenosis as assessed by CCTA and received coronary intervention immediately after CCTA, the intervention was not included in MACE as coronary revascularization in this study. Since patients in the CACS $>0$ group had more severe coronary atherosclerosis than those in the CACS $=0$ group, the incidence of MACE in the $\mathrm{CACS}>0$ group was naturally higher than that in the $\mathrm{CACS}=0$ group. Therefore, the main point in this study is that
CACS may be a very useful marker for predicting the prognosis.

\section{Study limitations}

This study has several important limitations. First, although severely impaired renal function is associated with a higher prevalence of CAD, such CAD patients may have been excluded because patients with creatinine $>2.0 \mathrm{mg} / \mathrm{dL}$ did not undergo CCTA. Second, MACE and the presence and severity of CAD were evaluated under various medications. A largescale prospective study will be needed to address these issues.

\section{Conclusions}

Only CACS $=0$ was independently associated with a lower
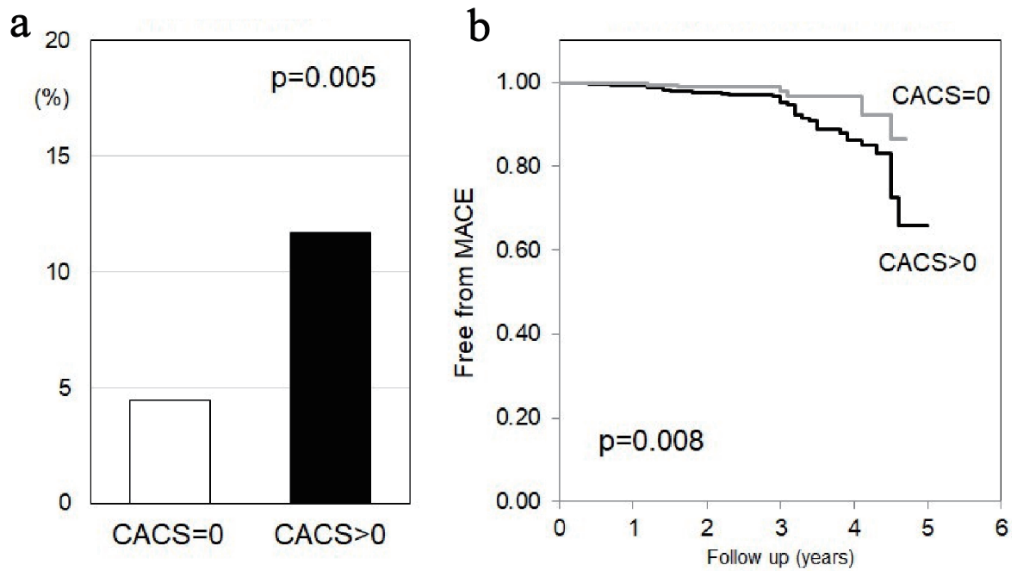

Figure 2. (a) \%MACE in the CACS $=0$ and CACS $>0$ groups. (b) Kaplan-Meier curves for freedom from MACE in the CACS $=0$ and CACS > 0 groups. MACE: major adverse cardiovascular events; CACS: coronary artery calcium score. 
Table 2. Independent Predictors of MACE by a Multivariate Logistic Regression Analysis

\begin{tabular}{lll} 
Factors & OR $(\mathbf{9 5} \% \mathbf{C I})$ & P value \\
\hline Age & $1.013(0.977-1.049)$ & 0.488 \\
Gender (male) & $2.091(0.923-4.734)$ & 0.077 \\
VFA & $1.001(0.995-1.007)$ & 0.821 \\
Smoking & $1.472(0.710-3.053)$ & 0.299 \\
Family history & $0.682(0.287-1.623)$ & 0.387 \\
HTN & $1.363(0.609-3.052)$ & 0.451 \\
DM & $0.973(0.462-2.048)$ & 0.941 \\
DL & $0.618(0.309-1.236)$ & 0.173 \\
CACS $=0$ & $0.405(0.170-0.965)$ & 0.041 \\
\hline
\end{tabular}

MACE: major adverse cardiovascular events; OR: odds ratio; Cl: confidence interval; VFA: visceral fat area; HTN: hypertension; DM: diabetes mellitus; DL: dyslipidemia; CACS: coronary artery calcium score.

incidence of MACE. Among all CVD risk markers, CACS of 0 was the strongest marker of a good prognosis.

\section{Acknowledgments}

We thank all of the members of the Department of Cardiology, Fukuoka University Hospital, and Department of Cardiology, Fukuoka University Nishijin Hospital, Fukuoka, Japan.

\section{Financial Disclosure}

None to declare.

\section{Conflict of Interest}

None to declare.

\section{Informed Consent}

All subjects gave their written informed consents

\section{Author Contributions}

Conceptualization: YS and SM. Validation: JM, KT, and YI. Formal analysis: YS, TK, and YY. Investigation: YS, JM, MS, and YK. Data curation: YS and KT. Writing of original draft: TK and MS. Writing of review and editing: SM. Supervision: SM.

\section{Data Availability}

The authors declare that data supporting the findings of this study are available within the article.

\section{References}

1. Detrano R, Guerci AD, Carr JJ, Bild DE, Burke G, Folsom $\mathrm{AR}$, Liu K, et al. Coronary calcium as a predictor of coronary events in four racial or ethnic groups. N Engl J Med. 2008;358(13):1336-1345.

2. Blaha MJ, Cainzos-Achirica M, Greenland P, McEvoy JW, Blankstein R, Budoff MJ, Dardari Z, et al. Role of coronary artery calcium score of zero and other negative risk markers for cardiovascular disease: the MultiEthnic Study of Atherosclerosis (MESA). Circulation. 2016;133(9):849-858.

3. Wang X, Le EPV, Rajani NK, Hudson-Peacock NJ, Pavey $\mathrm{H}$, Tarkin JM, Babar J, et al. A zero coronary artery calcium score in patients with stable chest pain is associated with a good prognosis, despite risk of non-calcified plaques. Open Heart. 2019;6(1):e000945.

4. Polonsky TS, McClelland RL, Jorgensen NW, Bild DE, Burke GL, Guerci AD, Greenland P. Coronary artery calcium score and risk classification for coronary heart disease prediction. JAMA. 2010;303(16):1610-1616.

5. Budoff MJ, Mayrhofer T, Ferencik M, Bittner D, Lee KL, Lu MT, Coles A, et al. Prognostic Value of Coronary Artery Calcium in the PROMISE Study (Prospective Multicenter Imaging Study for Evaluation of Chest Pain). Circulation. 2017;136(21):1993-2005.

6. Mitsutake R, Miura S, Kawamura A, Saku K. Are metabolic factors associated with coronary artery stenosis on MDCT? Circ J. 2009;73(1):132-138.

7. Mitsutake R, Miura S, Zhang B, Saku K. HDL-associated factors provide additional prognostic information for coronary artery disease as determined by multi-detector row computed tomography. Int J Cardiol. 2010;143(1):72-78.

8. Mitsutake R, Miura S, Shiga Y, Uehara Y, Saku K. Association between hypertension and coronary artery disease as assessed by coronary computed tomography. J Clin Hypertens (Greenwich). 2011;13(3):198-204.

9. Shiga Y, Miura S, Mitsutake R, Kawamura A, Uehara Y, Saku K. Significance of serum high-density lipoprotein cholesterol levels for diagnosis of coronary stenosis as determined by MDCT in patients with suspected coronary artery disease. J Atheroscler Thromb. 2010;17(8):870878.

10. Norimatsu K, Miura S, Suematsu Y, Shiga Y, Miyase Y, Nakamura A, Yamada M, et al. Associations between glycated albumin or hemoglobin A1c and the presence of coronary artery disease. J Cardiol. 2015;65(6):487-493.

11. Ueda Y, Shiga Y, Idemoto Y, Tashiro K, Motozato K, Koyoshi R, Kuwano T, et al. Association between the presence or severity of coronary artery disease and pericardial fat, paracardial fat, epicardial fat, visceral fat, and subcutaneous fat as assessed by multi-detector row computed tomography. Int Heart J. 2018;59(4):695704.

12. Gensini GG. A more meaningful scoring system for determining the severity of coronary heart disease. Am J Car- 
diol. 1983;51(3):606.

13. Agatston AS, Janowitz WR, Hildner FJ, Zusmer NR, Viamonte M, Jr., Detrano R. Quantification of coronary artery calcium using ultrafast computed tomography. J Am Coll Cardiol. 1990;15(4):827-832.
14. Kim YJ, Hur J, Lee HJ, Chang HJ, Nam JE, Hong YJ, Kim HY, et al. Meaning of zero coronary calcium score in symptomatic patients referred for coronary computed tomographic angiography. Eur Heart J Cardiovasc Imaging. 2012;13(9):776-785. 